\title{
Assessing the feasibility and acceptability of community health extension workers to treat hypertension associated with pregnancy: An analysis of post-intervention findings
}

Salisu Mohammed Ishaku

Population Council

Follow this and additional works at: https://knowledgecommons.popcouncil.org/departments_sbsr-rh

Part of the Civic and Community Engagement Commons, Demography, Population, and Ecology Commons, Family, Life Course, and Society Commons, and the International Public Health Commons How does access to this work benefit you? Let us know!

\section{Recommended Citation}

Ishaku, Salisu Mohammed. 2018. "Assessing the feasibility and acceptability of community health extension workers to treat hypertension associated with pregnancy: An analysis of post-intervention findings," Ending Eclampsia Research Brief. Abuja: Population Council. 


\section{ENDINC Eclampsia}

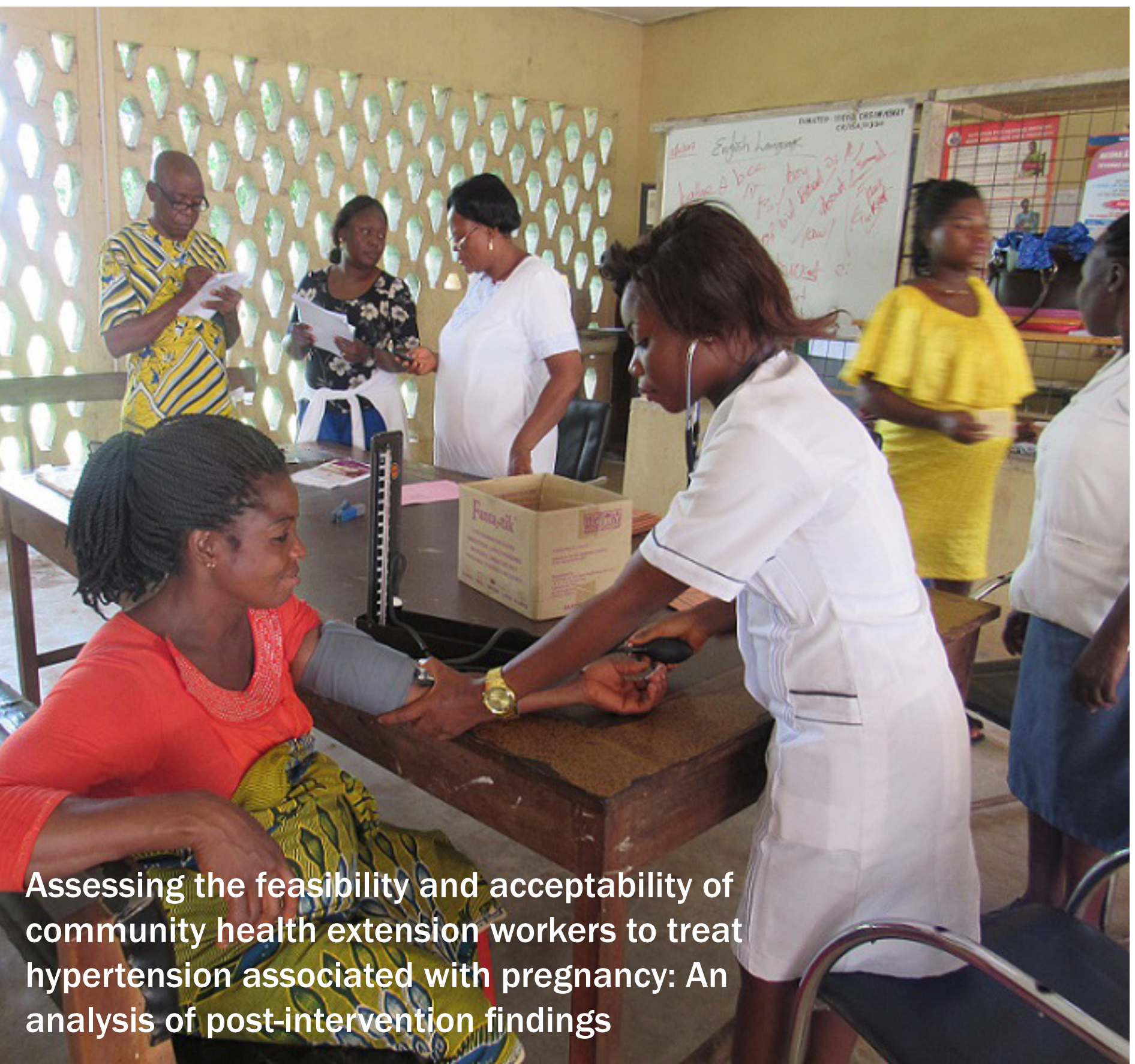

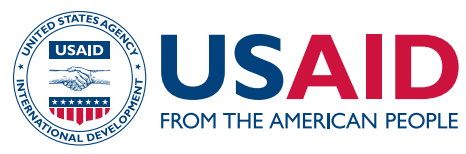

Made possible by the generous support of the American people through the United States Agency for International Development (USAID).
The Population Council conducts research and delivers solutions that improve lives around the world. Big ideas supported by evidence: It's our model for global change. popcouncil.org

(c) 2018 The Population Council, Inc

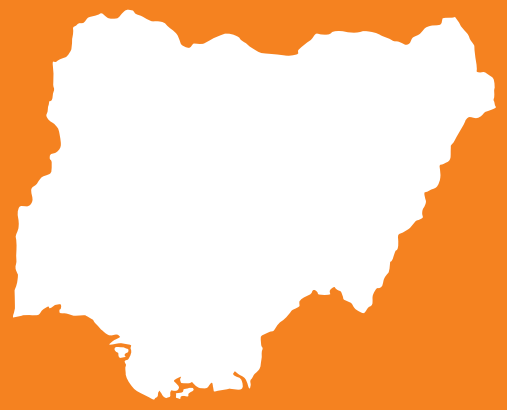

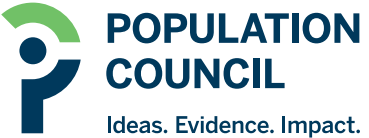




\section{BACKGROUND}

In Nigeria, hypertensive disorders of pregnancy (HDPs) - mainly pre-eclampsia and eclampsia (PE/E) - are the most common causes of death in pregnant women, accounting for 23 percent of maternal deaths in tertiary health facilities. Uncon $\neg$ trolled hypertension puts women at risk of cerebrovascular accident, a common cause of death from $\mathrm{PE} / \mathrm{E}$. Averting deaths from $\mathrm{PE} / \mathrm{E}$ requires early detection, timely delivery of the baby, effective use of antihypertensive drugs to control the associated hypertension, and administration of magnesium sulphate $\left(\mathrm{MgSO}_{4}\right)$ to prevent and treat convulsions.

Community Health Extension Workers (CHEWs) are authorized to administer the loading of $\mathrm{MgSO}_{4}$ prior to referring a pregnant woman with $\mathrm{PE} / \mathrm{E}$ to a secondary or tertiary health facility. Nigeria's task shifting policy is unclear on whether CHEWs - often the first points of contact for pregnant women - are permitted to administer antihypertensive drugs to pregnant women. There is a need, therefore, to determine whether CHEWs can safely administer antihypertensive drugs for HDPs.

\section{RESEARCH AIM}

This study tested the feasibility of tasking CHEWs with detecting and managing hypertension, and prescribing an oral antihypertensive drug alpha methyldopa (aldomet) - at primary health care (PHC) facilities as part of a package of care for women with PE/E. This evaluation measured improve $\neg$ ments in knowledge retention and increases in early detection, prevention, and management of $\mathrm{PE} / \mathrm{E}$.

\section{INTERVENTION}

In Ebonyi State, 43 CHEWs in 20 intervention PHCs received training (theory) and mentoring (skills) on accurate measurement of blood pressure (BP) in pregnant women and categorization as normal, mild, moderate, or severe hypertension, as well as how to manage hypertension using oral alpha methyldopa.

CHEWs in the intervention facilities prescribed and dispensed alpha methyldopa to women with moderate and se-vere hypertension. If the drug was not available at the facility, women and their families may have had to obtain the drug at outside, local pharmacies. CHEWs then asked women to return to the PHC for refills and BP measurement every month during pregnancy. If hypertension was not controlled, women were referred to a hospital. In an impending case of severe $\mathrm{PE} / \mathrm{E}$, the woman received the loading dose of $\mathrm{MgSO}_{4}$ according to national policy and was referred to a secondary facility for further management.

Twenty-nine CHEWs in 20 comparison facilities did not prescribe alpha methyldopa, but instead were expected to refer pregnant women with moderate or high BP to referral hospitals. Any available physicians in comparison facilities could dispense and prescribe antihypertensive drugs, based on their clinical judgment to best address the woman's condition.
Pre-eclampsia/ Eclampsia (PE/E) IN BRIEF

- Pre-eclampsia is a condition in pregnant women marked by an increase in blood pressure and protein in urine after 20 weeks gestation.

- $\quad$ Providing high quality antenatal care improves the prevention and early detection of pre-eclampsia and can prevent its progression to eclampsia.

- Eclampsia is a life-threatening condition characterized by convulsions in women with PE.

- Women in developing countries are 300 times more likely to die from eclampsia than women in developed countries.

- $\quad$ Prescribing low-dose aspirin and calcium to at-risk women can prevent pre-eclampsia and eclampsia.

- Pre-eclampsia and eclampsia can be managed by administering anti-hypertensive drugs and magnesium sulphate (MgSO4).

- MgSO4 is the safest and most effective treatment for severe $\mathrm{PE} / \mathrm{E}$, and is one of 13 UN LifeSaving Commodities for Women and Children.

- $\quad$ PE/E and other hypertensive disorders in pregnancy increase the risk of pre-term births, which can lead to low birth weight, anemia, and stunting.

- Improved prevention, increased detection, and effective treatment of PE/E can prevent unnecessary maternal and newborn deaths.
The Ending Eclampsia project seeks to expand access to proven, underutilized interventions and commodities for the prevention, early detection, and treatment of pre-eclampsia and eclampsia and strengthen global partnerships. 


\section{FINDINGS}

\section{CHEWs' Knowledge Retention}

Trained CHEWs working at intervention PHCs can correctly identify, differentiate, and manage normal, mild, moderate, and severe hypertension. At baseline, only $4 \%$ of intervention providers could correctly identify hypertension as normal, mild, moderate, or severe, but at endline $67 \%$ could correctly differentiate. Overall, intervention providers were $60 \%$ more likely to correctly identify hypertension than the comparison group $(p<.001)$. Following training, $75 \%$ providers could correctly describe how to manage hypertension, compared to only $40 \%$ at baseline $(p<0.001)$; comparison group providers saw a decrease in knowledge retention of hypertension management from 38\% to 16\% ( $p<0.001$ ). There was 55\% improvement in knowledge of proper timing for administration of a $\mathrm{MgSO}_{4}$ loading dose among trained providers, whereas comparison provider $\mathrm{MgSO}_{4}$ knowledge at endline improved by only 14\% $(p=0.021)$. Figure 1 below describes changes in CHEW's knowledge of HDPs pre- and post-intervention.

\section{CHEWs' Knowledge of Treating Hypertension}

Ninety-two percent of CHEWs in intervention facilities identified alpha methyldopa as a drug used to control BP at endline, compared to $14 \%$ at baseline. Overall, CHEWs in the intervention group were $36 \%$ more likely to name alpha methyldopa as a drug used to control blood pressure than CHEWs in the comparison group $(p=.019)$. In the intervention and control groups, CHEWs were more likely to prescribe alpha methyldopa over other antihypertensive drugs (60\% and 50\% of providers, respectively). Interestingly, theoretical knowledge of antihypertensives and actual practice do not always align and require sustained oversight and mentorship. For example, though almost no CHEWs in either the intervention or comparison groups spontaneously named Labetalol as a drug that controls blood pressure, in practice, it was the second most- prescribed antihypertensive drug after alpha methyldopa among CHEWs in the intervention group at endline (34.3\% of providers named it as the most prescribed antihypertensive at their facility).

\section{CHEWs' Ability to Diagnose \& Treat Hypertension}

Following training, only one patientseen by the intervention providers was incorrectly diagnosed, while one woman was correctly diagnosed with $\mathrm{PE}, 15$ with severe PE, and 23 with another HDP. Comparison providers incorrectly diagnosed nine women, but correctly diagnosed one woman with PE and four women with other HDPs.

Among women with moderate-to-severe hypertension who required antihypertensive drugs upon diagnosis, $89 \%$ of women in the intervention PHCs received treatment, compared to only $20 \%$ in the comparison PHCs. Furthermore, $50 \%$ of women with severe PE in the intervention $\mathrm{PHCs}$ received a loading dose of $\mathrm{MgSO}_{4}$ at diagnosis, but no women in the comparison PHCs received the loading dose. Most PHCs in intervention and comparison groups procure antihypertensive drugs from facility arrangements within the open market $(92 \%$ and $57 \%$, respectively). CHEWs from both groups reported that procurement cost is the greatest barrier to stocking antihypertensive drugs.

Between July 2016 and December 2017, 54 pregnant women from Ebonyi State who presented with high BP were monitored and followed up until after delivery. See Table 1 for client details. Gestational age at point of first contact with PHCs shows that no women in our sample sought ANC prior to 12 weeks of pregnancy and many waited until close to their third trimester, suggesting normalized delays maternal care-seeking.

\section{Maternal \& Fetal Health Outcomes}

Throughout the duration of this study, there were five maternal deaths - one in the intervention group and four in the comparison group. Thirteen fetal deaths occurred, four in the intervention group and nine in the

Figure 1: CHEWs' pre- and post-intervention knowledge of hypertensive disorders in pregnancy

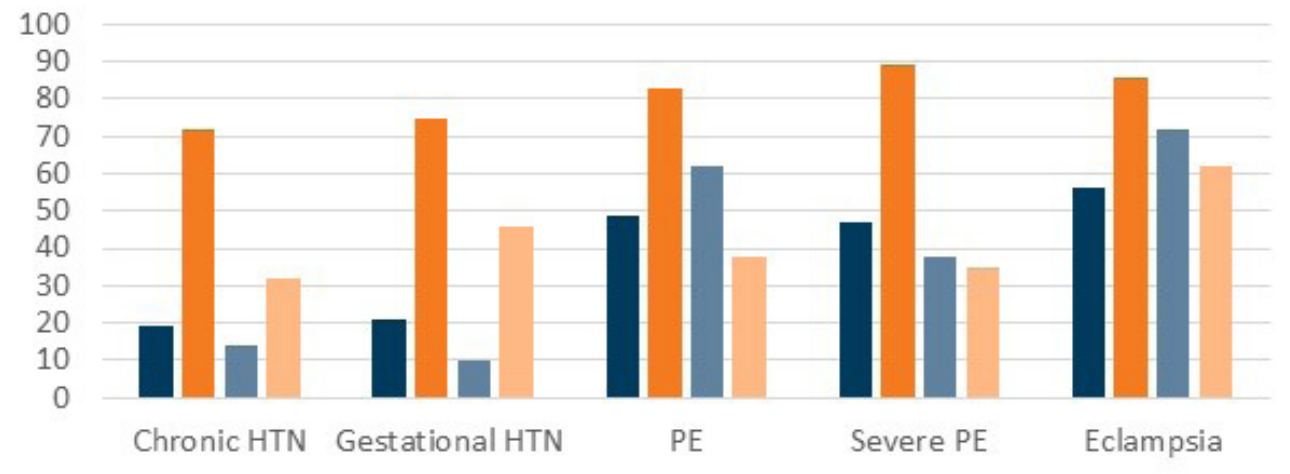


Table 1: Characteristics of pregnant women at first and follow up ANC visits

\begin{tabular}{|c|c|c|c|c|}
\hline \multirow[t]{3}{*}{ Age } & $15-24$ & $9(22.5)$ & $5(35.7)$ & $14(25.9)$ \\
\hline & $25-34$ & $19(47.5)$ & $4(28.6)$ & $23(42.6)$ \\
\hline & $>35$ & $12(30)$ & $5(35.7)$ & $17(31.5)$ \\
\hline \multirow[t]{4}{*}{ Education } & o formal education & $7(17.5)$ & $1(7.7)$ & $8(15.1)$ \\
\hline & Primary & $11(27.5)$ & $4(30.8)$ & $15(28.3)$ \\
\hline & Secondary & 19 (47.5) & $8(61.6)$ & $27(50.9)$ \\
\hline & First degree & $3(7.5)$ & 0 & $3(5.6)$ \\
\hline \multirow[t]{3}{*}{ BP at ANC booking } & $<140 / 90$ mmhg & $14(35)$ & $10(71.4)$ & $24(44.4)$ \\
\hline & $>140 / 90 \mathrm{mmhg}$ & $21(52.5)$ & $2(14.3)$ & $23(42.6)$ \\
\hline & Unrecorded & $5(12.5)$ & $2(14.3)$ & $7(13)$ \\
\hline \multirow[t]{4}{*}{ Proteinuria at ANC booking } & +1 & 0 & 0 & 0 \\
\hline & +2 & $2(10)$ & 0 & $4(7.4)$ \\
\hline & $>+3$ & $5(12.5)$ & 0 & $5(9.3)$ \\
\hline & Nil/ trace & $31(77.5)$ & $14(100)$ & 45 (83.3) \\
\hline \multirow[t]{4}{*}{ Gestation at ANC booking } & $<12$ weeks & 0 & 0 & 0 \\
\hline & $12-20$ weeks & $13(32.5)$ & $5(35.7)$ & 18 (33.3) \\
\hline & 21-30 weeks & $11(27.5)$ & $4(28.6)$ & $15(28)$ \\
\hline & $31-40$ weeks & $10(25)$ & $2(14.3)$ & $12(19.7)$ \\
\hline \multirow[t]{4}{*}{ Gestation at HDP diagnosis } & $<12$ weeks & 0 & 0 & 0 \\
\hline & $12-20$ weeks & $6(15)$ & $3(21.4)$ & $9(16.7)$ \\
\hline & 21-30 weeks & 15 (37.5) & $4(28.5)$ & $19(35.2)$ \\
\hline & $31-40$ weeks & $15(37.5)$ & $7(50)$ & $22(40.8)$ \\
\hline
\end{tabular}

comparison group. All maternal deaths occurred among women who did not receive $\mathrm{MgSO}_{4}$. To better understand barriers that lead to these deaths, further analysis of the timing of severe PE/E diagnosis and reasons for delay in administering the loading dose is needed.

\section{DISCUSSION}

Findings from this study to task-shift PE/E detection and management to CHEWs are encouraging. Overall, PHC providers are able to distinguish mild, moderate, and severe hypertension in pregnant women and diagnose PE/E at PHCs. Improvements among CHEWs who received intensive training, as well as differences between intervention and comparison CHEWs, indicate that HDPs and PE/E can be detected and managed at the PHC level. Challenges continue when ensuring women have access to care early in their pregnancies. As mentioned, appropriate PE/E management requires early detection, but the majority of women came to their first ANC appointment (booking appointment) once high BP had occurred and they felt unwell.

A national task-shifting policy exists in Nigeria, and these findings are important contributions to implementing the national policy at the local level. Training and mentoring CHEWs to detect and manage PE/E at the local level will improve access to life-saving care for women, their families, and their communities, and will contribute to stronger health systems and improved maternal health outcomes in Nigeria.

\section{RECOMMENDATIONS}

- Sustain CHEW training, mentoring, and oversight, as needed for the detection and management of HDPs;

- $\quad$ Streamline state procurement for supplies and commodities, and enhance linkages to central distribution system of antihypertensive drugs and $\mathrm{MgSO}_{4}$;

- Amend national task-shifting policy to explicitly include antihypertensive drug administration at the PHC level;

- $\quad$ Scale up task-shifting for antihypertensive drug use at PHC level across Nigeria; and

- $\quad$ Conduct further analysis to understand women's maternal health-seeking behavior.

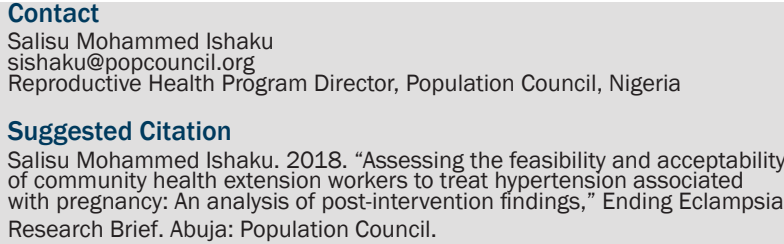

